\title{
On the specificity of avian blood parasites: revealing specific and generalist relationships between haemosporidians and biting midges
}

\author{
JOSUÉ MARTÍNEZ-de LA PUENTE, ${ }^{* 1}$ JAVIER MARTÍNEZ,† JUAN RIVERO-de AGUILAR, *† \\ JESSICA HERRERO† and SANTIAGO MERINO* \\ *Departamento de Ecología Evolutiva, Museo Nacional de Ciencias Naturales (CSIC), J. Gutiérrez Abascal 2, E-28006 Madrid, \\ Spain, †Departamento de Microbiología y Parasitología, Facultad de Farmacia, Universidad de Alcalá, Alcalá de Henares, \\ E-28871 Madrid, Spain
}

\begin{abstract}
The study of host-parasite relationships involving vector-borne parasites requires understanding interactions between parasites and vectors. The capacity of haemosporidians to infect insects has clear evolutionary consequences for the transmission of diseases. Here, we investigated (i) the associations between blood parasites, biting midges and birds and (ii) the potential specificity between biting midge and haemosporidian haplotypes. A total of 629 parous biting midges Culicoides and 224 wild birds (belonging to seven species) from a locality of central Spain were individually examined for the presence of Haemoproteus and Plasmodium parasites by sequencing a fragment of cytochrome B. Biting midges were identified morphologically and characterized on the basis of a fragment of the cytochrome c oxidase (COI) gene. Overall, 12 Haemoproteus and three Plasmodium haplotypes were isolated and sequenced. Among them, 10 haplotypes were exclusively isolated from biting midges, three haplotypes only from birds and two haplotypes from both biting midges and birds. Biting midge haplotypes showed both specific and generalist relationships with Haemoproteus haplotypes but only generalist relationships with Plasmodium haplotypes. Several C. festivipennis and C. kibunesis haplotypes established significant coevolutionary links with Haemoproteus haplotypes. These results shed light on the specificity of interactions between vectors and blood parasites.
\end{abstract}

Keywords: avian malaria, blue tits Cyanistes caeruleus, Culicoides, Haemoproteus, host-parasite interactions

\section{Introduction}

Haemosporidians are obligate parasites that infect a wide range of vertebrates and use dipteran insects as vectors (where sexual reproduction takes place). Avian malaria parasites and related haemosporidians include blood parasites belonging to the genera Plasmodium,

Correspondence: Josué Martínez-de la Puente, Fax: +34 954 621 125; E-mail: jmp@ebd.csic.es

${ }^{1}$ Present address: Departamento de Ecología de Humedales, Estación Biológica de Doñana (CSIC), Américo Vespucio s/n, E-41013, Sevilla, Spain.
Haemoproteus and Leucocytozoon (Valkiūnas 2005). These parasites impact the evolution of their hosts through detrimental effects on their reproductive success and survival probability (Merino et al. 2000; Valkiūnas \& Iezhova 2004; Martínez-de la Puente et al. 2010).

The ability of haemosporidians to infect vertebrate hosts and insects has clear evolutionary consequences for the transmission of diseases in the wild (i.e. Gager et al. 2008; Hellgren et al. 2008; Ishtiaq et al. 2008). At least two main possible roles for vectors in blood parasite transmission between avian species have been suggested. First, vectors eating blood mainly or 
exclusively from a limited number of host species (Malmqvist et al. 2004) could limit the spread of blood parasites (Hellgren et al. 2008). Alternatively, vectors could feed blood on a wide range of avian species (Gager et al. 2008; Kim et al. 2009) and, if the parasite is competent to develop sexual reproduction and producing infective forms in the vector, thereby contribute to the spread of parasite haplotypes between birds. Thus, parasites capable of being transmitted by a wide range of insect vectors (vector-generalist parasites) or even by host-generalist ones may attain higher transmission success.

Among avian malaria parasites and related haemosporidians, comparison of host and parasite phylogenies has revealed different degrees of host specialization between parasite genera (Beadell et al. 2004). However, this information is clearly biased towards studies conducted on vertebrate hosts. Currently, data on the level of vector-parasite specificity are scarce despite its importance for avian malaria epidemiology. Traditionally, Plasmodium and Haemoproteus have been considered vector-generalist parasites (Valkiūnas 2005). However, although Plasmodium parasites are capable of being transmitted by insects belonging to different genera (Ejiri et al. 2008; Ishtiaq et al. 2008; Kimura et al. 2010; but see Gager et al. 2008), the transmission of Haemoproteus (subgenus Parahaemoproteus) is restricted to biting midges belonging to the genus Culicoides (Valkiūnas 2005) suggesting that both parasite genera could vary in their degree of insect specialization. By contrast, Ishtiaq et al. (2008) found a general lack of cospeciation for both Plasmodium and Haemoproteus in mosquitoes (see also Njabo et al. 2011). However, because (i) the capacity to transmit a particular blood parasite species could vary between insect species and even among individuals within the same species (Lambrechts et al. 2005) and (ii) host specificity is highly linked with cospeciation processes (Poulin 1992; Kearn 1994), important questions remain over the specific associations between insect and parasite haplotypes.

Biting midges Culicoides are vectors of different haemosporidians including Haemoproteus, Leucocytozoon (subgenus Akiba) and Hepatocystis (Fallis \& Wood 1957; Garnham et al. 1961; Valkiūnas 2005). Also, the reptilian malaria parasite Plasmodium agamae completes development in a Culicoides species (Petit et al. 1983). Previous studies have been conducted on mosquitoes and black flies transmitting mainly Plasmodium and Leucocytozoon parasites (Ejiri et al. 2008; Gager et al. 2008; Hellgren et al. 2008; Ishtiaq et al. 2008; Kim et al. 2009; Kimura et al. 2010). However, it is still necessary to identify the role of biting midges in parasite-vector-host associations in the wild because Haemoproteus parasites are considered to be exclusively transmitted by biting midges and louse flies (Valkiūnas 2005), and the ecology and biting behaviour of mosquitoes, black flies and biting midges differ substantially.

Here, we identified the Haemoproteus and Plasmodium haplotypes shared by biting midges and wild birds to: (i) explore the specificity of parasite haplotypes in avian species, particularly with respect to testing whether Haemoproteus parasites are actually specific at the bird family level and whether Plasmodium parasites are generalists and (ii) explore the degree of specificity of parasite haplotypes in biting midges given that they are considered vectors of Haemoproteus but not of Plasmodium. We conducted our study on individual parous wild-caught biting midges and on blood samples from wild birds. We also explored whether the associations between parasite and insect haplotypes show evidence of cospeciation. Knowledge of these relationships will throw light on the associations between parasite haplotypes and vectors in the wild and may have important implications for the evolution of virulence in blood parasites (Ewald 1994).

\section{Material and methods}

This study was carried out in a Pyrenean oak Quercus pyrenaica deciduous forest located in Valsaín (Central Spain, $40^{\circ} 53674 \mathrm{~N}, 4^{\circ} 01 \mathrm{cW}, 1200 \mathrm{~m}$ a.s.l.). During the spring of 2006, we captured biting midge Culicoides in blue tit Cyanistes caeruleus nests using nest-box traps (see Tomá s et al. 2008 for a description of the method). Biting midges were removed from capture dishes with xylene and preserved in absolute ethanol until their identification. Later, biting midges were transferred to $70 \%$ ethanol, sexed and the parity of females determined as follows: nulliparous (those that have never fed on blood), parous (see below) or engorged females (those with a bloodmeal still not completely digested in their abdomen). Parous females were identified by the burgundy pigment present in the subcutaneous cells of the abdomen, indicating they had digested a bloodmeal prior to capture (Dyce 1969). The method described by Dyce (1969) has been used to perform studies on the identification of viral infections (Nelson \& Scrivani 1972) and Haemoproteus transmission infectivity (Mullens et al. 2006). Parous biting midges look for a new bloodmeal after the first gonotrophic cycle is completed (about 48-72 h after mating; Marquardt et al. 2000) and therefore they are the only ones likely to yield blood parasite sporozoites. Parous biting midges were morphologically identified according to their wing pattern under an Olympus SZH stereomicroscope (10-64- magnification) using available keys (Kremer 1966; Delécolle 1985). 


\section{Molecular characterization of biting midges}

Parous biting midges preserved in $70 \%$ ethanol were individually crushed on filter paper discs (diameter $5 \mathrm{~mm}$ ) with a metal rod. Genomic DNA from dried samples was extracted using the following protocol: the discs were immersed in $250 \mathrm{lL}$ of SET buffer $(0.15 \mathrm{M}$ $\mathrm{NaCl}, 50 \mathrm{~mm}$ Tris, $1 \mathrm{~mm}$ EDTA, $\mathrm{pH}=8$ ) and SDS $20 \%$ (7 1L) and proteinase K (50 Ig) were immediately added to the vials, maintaining the mix in a incubating shaker at $55^{\circ} \mathrm{C}$ overnight. The following day, ammonium acetate $4 \mathrm{~m}(250 \mathrm{IL})$ was added to the vials at room temperature for $30 \mathrm{~min}$. Subsequently, vials were centrifuged at $13000 \mathrm{~g}$ for $10 \mathrm{~min}$. After removing the pellet, DNA was precipitated with ethanol, washed with ethanol $70 \%$ and resuspended in sterile water.

Molecular characterization of biting midges harbouring blood parasites was conducted. A 472-bp segment of the mitochondrial cytochrome c oxidase subunit I (COI) gene was amplified using primers C1-J-1718 (5 GGA TTT GGA AAT TGA TTA GTT CC-3c) and C1-N2191 (5-CCC GGT AAA ATT AAA ATA TAA ACT TC3c) (Dallas et al. 2003). Polymerase chain reactions (PCRs) consisted of 25-1L reaction volumes containing 20-ng template DNA, $50 \mathrm{~mm} \mathrm{KCl,} 10 \mathrm{~mm}$ Tris- $\mathrm{HCl}, 1.5$ $\mathrm{MgCl}_{2}, 0.2 \mathrm{~mm}$ of each dNTP, $0.5 \mathbf{l}_{\mathrm{M}}$ of each primer and $1.25 \mathrm{U}$ of AmpliTaq Gold (Applied Biosystems, Foster City, CA, USA). The reactions were cycled at the following parameters using the thermal cycler 2720 (Applied Biosystems): $94{ }^{\circ} \mathrm{C}$ for $10 \mathrm{~min}$ (polymerase activation), 40 cycles at $95{ }^{\circ} \mathrm{C}$ for $40 \mathrm{~s}$ (denaturing), $52{ }^{\circ} \mathrm{C}$ for $1 \mathrm{~min}$ (annealing temperature), $72{ }^{\circ} \mathrm{C}$ for $1 \mathrm{~min}$ (extension) and a final extension at $72{ }^{\circ} \mathrm{C}$ for $10 \mathrm{~min}$. Amplicons obtained after PCR assays were recovered from agarose gels and sequenced using an ABI 3130 (Applied Biosystems) automated sequencer. Additional samples from each morphospecies were analysed to confirm the morphological identifications and characterize the genetic diversity within each morphospecies.

\section{Blood parasite detection in biting midges}

We detected Haemoproteus and Plasmodium by amplifying a 390-bp fragment of the mitochondrial cytochrome $B$ gene using the primers Palu-F (5)-GGG TCA AAT GAG TTT CTG G-3c) and Palu-R (5c-DGG AAC AAT ATG TAR AGG AGT-3c) (Martínez et al. 2009). PCRs consisted of 25-1L reaction volumes containing between 20- and 100-ng template DNA, $50 \mathrm{~mm} \mathrm{KCl,} 10 \mathrm{~mm}$ Tris$\mathrm{HCl}, 1.5 \mathrm{MgCl}_{2}, 0.2 \mathrm{~mm}$ of each dNTP, $0.5 \mathbf{l} \mathrm{M}$ of each primer and $1.25 \mathrm{U}$ of AmpliTaq Gold (Applied Biosystems). The reactions were cycled at the following parameters: $94^{\circ} \mathrm{C}$ for $10 \mathrm{~min}$ (polymerase activation), 40 cycles at $95{ }^{\circ} \mathrm{C}$ for $40 \mathrm{~s}$ (denaturing), $54^{\circ} \mathrm{C}$ for $1 \mathrm{~min}$ (annealing temperature), $72^{\circ} \mathrm{C}$ for 1 min (extension) and a final extension at $72{ }^{\circ} \mathrm{C}$ for $10 \mathrm{~min}$. Amplicons obtained after PCR assays were recovered from agarose gels and sequenced using an ABI 3130 (Applied Biosystems) automated sequencer. To prevent contamination, we used different sets of pipettes and filter tips for extraction, PCR set up and downstream fragment analyses. We never amplified DNA from negative controls added in each PCR batch.

\section{Blood parasite detection in birds}

During the springs of 2005-2009, we sampled blood from blue tits Cyanistes caeruleus (34 adults), great tits Parus major (61 adults and 20 nestlings), coal tits Periparus ater (one adult and eight nestlings), pied flycatchers Ficedula hypoleuca (23 adults), eurasian nuthatches Sitta europaea (22 adults and 47 nestlings), rock sparrows Petronia petronia (two adults and four nestlings) and great spotted woodpeckers Dendrocopos major (two adults) breeding in the study area.

Blood samples were stored on FTA classic cards (Whatman International Ltd., UK). Genomic DNA was extracted to a soluble solution using the protocol previously described for biting midges. The partial amplifications of the Haemoproteus and Plasmodium cytochrome B gene were accomplished using the PCR protocol described above. We assume that records of parasite DNA in birds are indicative of infections, note that this does not necessarily mean that the parasites complete their development in birds (as shown in Leucocytozoon infections; Valkiūnas et al. 2009).

\section{Phylogenetic analysis}

For biting midges, the 39 haplotypes obtained here were aligned along with other 33 biting midge sequences listed in GenBank. Overall, the alignment contained sequences corresponding to 31 morphospecies. The alignment was performed using the CLUSTALW algorithm implemented in BIOEDIT program (Hall 1999). The minimum number of sequences for the flank positions of the alignment was 50\%, and it was performed with the program Gblocks (Castresana 2000; Talavera \& Castresana 2007). The final alignment contained 472 positions and 74 sequences including two sequences corresponding to Simulium rufibasis and S. bidentatum (GenBank accession numbers DQ534950 and DQ534946, respectively) as an outgroup.

The Haemoproteus and Plasmodium haplotypes isolated from biting midges and birds were aligned together with the other sequences listed in GenBank (see Results). Some sequences with identical overlapping DNA fragments were maintained in the alignment if 
they contained a tail (3cor 5tails) that was present in only one sequence (e.g. CulHae4 and GAGLA03 or SePlas1 and Padom5). The DNA sequences were aligned using the CLUSTALW algorithm implemented in BIOEDIT (Hall 1999). The original alignment contained $7306 \mathrm{bp}$, but it was delimited at 3c extreme by the final of the sequences corresponding to the haplotypes isolated in the present study. However, we maintained a 5 tail containing 416 bp because it was present in more than half of the sequences in the alignment. Consequently, the final alignment contained 768 positions and 97 taxa, including two sequences of Leucocytozoon as an outgroup.

The alignments were analysed using Bayesian inferences implemented in the program MrBayes v3.2 (Ronquist \& Huelsenbeck 2003) setting the substitution model HKY for biting midges and GTR for blood parasites. The models were previously selected using corrected AIC implemented in jModeltest 0.0.1 (Posada 2008). The parameter gamma shape and the proportion of invariable sites were estimated by the program ('rates' was set as 'invgamma'). Metropolis-coupled Markov chain Monte Carlo (MCMCMC) analyses were run for 20000000 generations and sampled every 1000 generations. The rest of the parameters were set at their default options. At the end of the analysis, we set the burn-in period to $50 \%$ where the chains reached stationary phase (standard deviation <0.01). Nevertheless, the convergence of the parameters and topology was tested using the TRACER (Rambaut \& Drummond 2007) and AWTY (Wilgenbusch et al. 2004; Nylander et al. 2008) applications, respectively. In both cases, the statistical data and graphs did not show lack of convergence.

\section{Estimates of evolutionary divergence}

Estimates of evolutionary divergence over sequences were conducted using the uncorrected p-distance method with the option pairwise deletion implemented in MEGA4 (Tamura et al. 2007).

Statistical analyses for biting midge-haemosporidian coevolution

The statistical method ParaFit (Legendre et al. 2002) implemented in CopyCat software (Meier-Kolthoff et al. 2007) was used to test the significance of a global hypothesis of coevolution between biting midge and haemosporidian haplotypes isolated in this study. ParaFit method conducts a statistical test for the presence of congruence between host and parasite phylogenies but also each host-parasite link is assessed separately for its fit to the coevolution hypothesis. The global null hypothesis is that evolution of the hosts and parasites has been independent. To perform the test, the biting midge and haemosporidian phylogenetic trees with haplotypes identified here were constructed using Bayesian inference implemented in MrBayes v3.2 (Ronquist \& Huelsenbeck 2003). The GTR substitution model was selected for both trees using corrected AIC implemented in jModeltest, version 0.0.1 (Posada 2008). The rest of parameters were identical to those described in the previous section except for the number of generations, in this case was 5000 000. The individual hostparasite association links were extracted from the Table 1. Maximum Likelihood (PhyML software) and Neighbor-Joining (MEGA 4.0 software) trees were used to corroborate the results obtained.

\section{Results}

Biting midge species composition and molecular identification

A total of 629 parous biting midges were individually identified to morphospecies based on morphology. Overall, 39 different haplotypes from 93 biting midges (including those positive for blood parasite infection; see Table 1) were found. Six haplotypes were obtained from flies morphologically identified as Culicoides circumscriptus (11 sampled individuals), 12 from C. festivipennis (14 sampled individuals), five from C. kibunensis (15 sampled individuals), two from C. pictipennis (five sampled individuals), three from C. segnis (17 sampled individuals), 10 from C. simulator (24 sampled individuals) and one from C. truncorum (seven sampled individuals). The highest phylogenetic divergence of the genetic haplotypes was found for C. pictipennis and C. segnis $(7.9 \%$ and $7.7 \%$, respectively) followed by C. simulator (1.1\%), C. festivipennis (1\%), C. kibunensis $(0.7 \%)$, C. circumscriptus $(0.5 \%)$ and C. truncorum (presented a single haplotype). Genetic haplotypes obtained from each morphospecies captured here formed wellsupported monophyletic clades (Fig. 1).

\section{Blood parasite diversity in biting midges and birds}

A total of 629 parous biting midges were individually tested for the presence of Haemoproteus and Plasmodium parasites (see Table 1). Overall, 15 different haemosporidian haplotypes were isolated from biting midges and birds. Among them, 10 haplotypes were exclusively found in biting midges (CulHae2 to CulHae10 corresponding to Haemoproteus and CulPlas2 to Plasmodium), three haplotypes only in birds (Haemoproteus haplotypes FhHae1 and FhHae2 in pied flycatchers F. hypoleuca and the Plasmodium haplotype SePlas1 in eurasian nuthatches 
Table 1 Plasmodium and Haemoproteus haplotypes isolated from biting midge morphospecies sampled at the same study area. The number of times a haplotype is detected is shown, and the biting midge haplotypes infected by each blood parasite haplotype is indicated between brackets. Biting midge morphospecies sampled in the present study were Culicoides circumscriptus (C), C. festivipennis (FV), C. kibunensis (K), C. pictipennis (P), C. segnis (SG), C. simulator (S) and C. truncorum (T)

\begin{tabular}{|c|c|c|c|c|c|c|c|c|}
\hline \multirow[b]{2}{*}{ Parasite haplotypes } & \multicolumn{8}{|c|}{ Culicoides morphospecies } \\
\hline & $\mathrm{C}$ & $\mathrm{FV}$ & $\mathrm{K}$ & $\mathrm{P}$ & SG & $\mathrm{S}$ & $\mathrm{T}$ & Total \\
\hline CulHae1‡ & 2 (C6) & & $3(\mathrm{~K} 1, \mathrm{~K} 4)$ & & & $2(\mathrm{~S} 1, \mathrm{~S} 4)$ & $1(\mathrm{~T} 1)$ & 8 \\
\hline CulHae2 & & & $1(\mathrm{~K} 1)$ & $1 \dagger$ & & 1 (S4) & & 3 \\
\hline CulHae3 & & & 1 (K5) & & & & & 1 \\
\hline CulHae4 & $2(\mathrm{C} 5)$ & 1 (FV10) & & $1 \dagger$ & & 3 (S5, S9) & & 7 \\
\hline CulHae5 & & & & & & $1(\mathrm{~S} 4)$ & & 1 \\
\hline CulHae6 & & & & $1 \dagger$ & & & & 1 \\
\hline CulHae7 & & & & & 1 (SG1) & & & 1 \\
\hline CulHae8 & & & & & & $1(\mathrm{~S} 5)$ & & 1 \\
\hline CulHae9 & $1(\mathrm{C} 2)$ & & & & & $2(\mathrm{~S} 4, \mathrm{~S} 6)$ & & 3 \\
\hline CulHae10 & & 2 (FV11, FV12) & & & & & & 2 \\
\hline CulPlas1‡ & & $1 \dagger$ & $2(\mathrm{~K} 2)$ & $3(\mathrm{P} 1)$ & & $3(\mathrm{~S} 5, \mathrm{~S} 6)$ & $2(\mathrm{~T} 1)$ & 11 \\
\hline CulPlas2 & & $1 \dagger$ & & & & & & 1 \\
\hline Infected flies & 5 & 5 & 7 & 6 & 1 & 13 & 3 & 40 \\
\hline Total sampled & 30 & 158 & 218 & 16 & 27 & 173 & 7 & 629 \\
\hline Prevalence & 16.7 & 3.2 & 3.2 & 37.5 & 3.7 & 7.5 & 42.9 & 6.4 \\
\hline
\end{tabular}

†Molecular characterization failed.

¥Blood parasite haplotypes also isolated from avian species breeding in the study area.

S. europaea) and two haplotypes were found in both biting midges and birds (CulHae1 Haemoproteus haplotype in blue tits C. caeruleus, great tits P. major and eurasian nuthatches S. europaea and CulPlas1 Plasmodium haplotype in blue tits C. caeruleus, great tits P. major, pied flycatchers F. hypoleuca, coal tits P. ater, eurasian nuthatches S. europaea and rock sparrows P. petronia). The mean uncorrected p-distance between haplotypes was $4.4 \%$ (range: $0.3-6.9 \%$ ) and $1.6 \%$ (range: $0.6-2.2 \%$ ) for Haemoproteus and Plasmodium, respectively.

Haemoproteus and Plasmodium parasites were found in $28(4.4 \%)$ and 12 (1.9\%) biting midges, respectively (Table 1). On average, each Haemoproteus and Plasmodium haplotype was present in 1.9 and 3 biting midge morphospecies, respectively.

Phylogenetic relationships of haemosporidians found in biting midges and birds

The phylogenetic tree including the haemosporidian haplotypes isolated from biting midges and birds is shown in Fig. 2. Some of the haplotypes recovered in this study correspond with haplotypes previously deposited in Genbank. CulHae2 and CulHae3 haplotypes probably belong to the same species because the genetic distance between both haplotypes is very low (0.3\%). In general, Haemoproteus haplotypes infecting birds from the same superfamilies were clustered. However, this is not the case for Plasmodium haplotypes.
Statistical analyses for biting midge-haemosporidian coevolution

The result of the global test of coevolution between biting midge and haemosporidian haplotypes was not significant $(P=0.075)$. Similar results were obtained using neighbour-joining $(\mathrm{P}=0.131)$ or maximum-likelihood $(\mathrm{P}=0.072)$ methods to obtain the phylogenetic trees. However, eight out of a total of 26 biting midge-haemosporidian links were significant. When the global test is not significant, it is recommended to take into account only highly significant links to compensate for the fact that the tests of individual links have inflated type I error (Meier-Kolthoff et al. 2007). Thus, we only selected the three links with $\mathrm{P}<0.01$ (CulHae10/FV11, CulHae10/FV12 and CulHae4/FV10) as those significantly showing host-parasite coevolution. Only these three associations were also significant at $\mathrm{P}<0.01$ using maximum-likelihood or neighbour-joining trees in the analysis (Table 2).

Because parasites belonging to Haemoproteus and Plasmodium may vary in the degree of host specificity affecting the results obtained here, we assessed the consistency of the phylogenetic trees using only the Haemoproteus haplotypes and reanalysed their coevolutionary relationships. In this case, the global test of coevolution was significant using the three sets of trees (Bayesian inference, $\mathrm{P}=0.019$; maximum likelihood, $\mathrm{P}=0.006$ and neighbour joining, $\mathrm{P}=0.006$ ). As the global test 


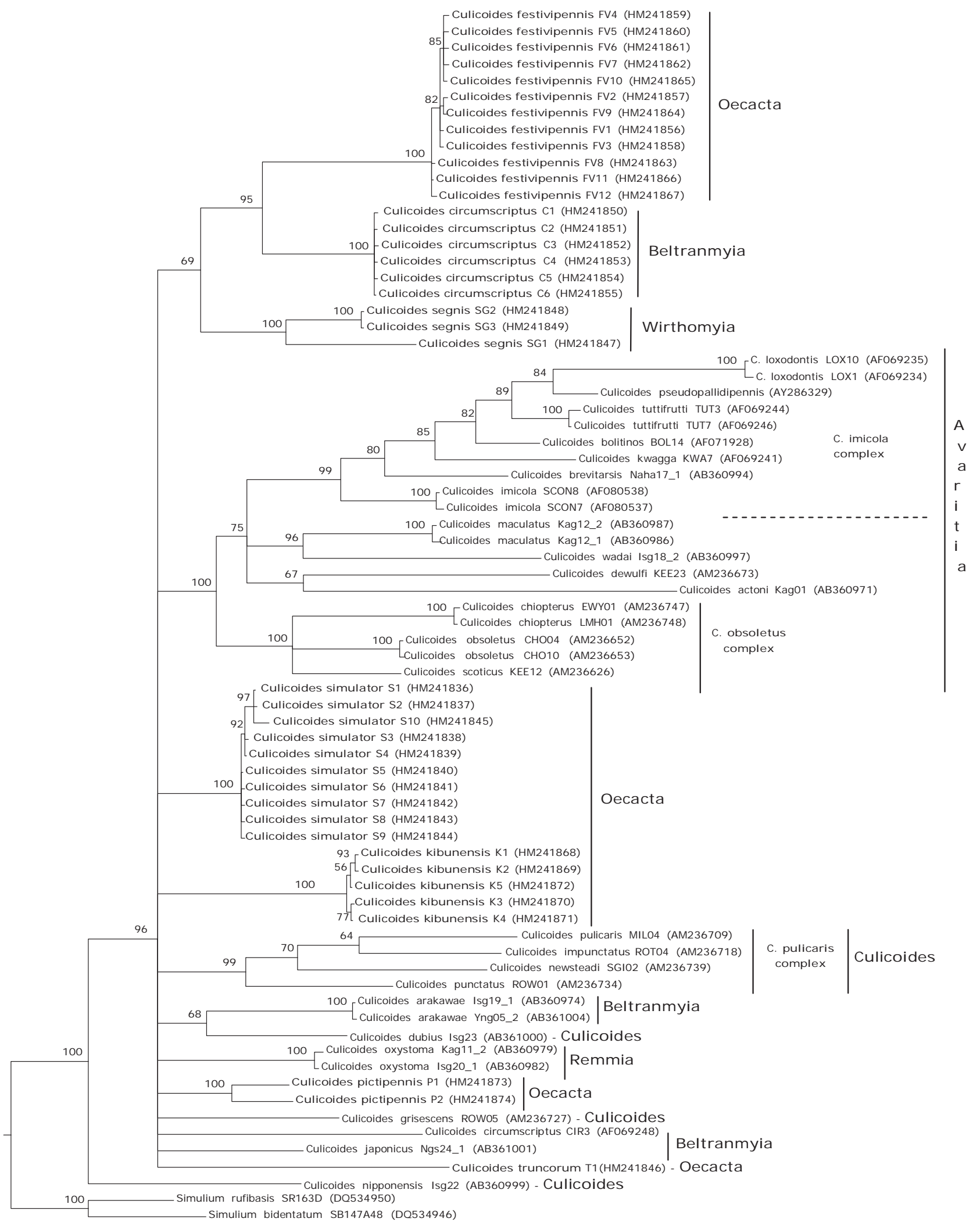


was significant, we did not establish a high significance threshold to select the representative host-parasite associations. In addition to the three significant host-parasite associations previously found, four new significant individual host-parasite associations arose using any of the phylogenetic methods (CulHae1/K1, CulHae1/K4, CulHae2/K1 and CulHae3/K5). Overall, seven host-parasite links showed a significant coevolutionary relationship involving two biting midge morphospecies, C. festivipennis (FV10, FV11 and FV12) and C. kibunensis (K1, K4 and K5), and five Haemoproteus haplotypes, two of them belonging to a known species (CulHae1 = $\mathrm{H}$. majoris, CulHae $2=\mathrm{H}$. minutus) and the other three to unknown species (CulHae 10, CulHae3 and CulHae4) (Table 3; Fig. 3).

\section{Discussion}

\section{Identification of biting midges}

Haplotypes from each biting midge morphospecies formed well-supported monophyletic clades in the phylogeny, supporting the congruence between the morphological identification and molecular characterization. Surprisingly, a sequence corresponding to a Culicoides circumscriptus captured in Portugal (AF069248) does not group together with those haplotypes isolated here. High intraspecific differences between populations or a misidentification of the fly could explain this incongruence. Differences among haplotypes within each morphospecies ranged between $0.5 \%$ and $1.1 \%$, with the exception of C. segnis and C. pictipennis, which had divergence values of $7.8 \%$ and $7.9 \%$, respectively. These higher values could be indicative of the presence of cryptic species because similar values are found when we estimated this parameter on groups formed by genetic haplotypes from two or more species. In this respect, it could be possible that C. reconditus and /or C. riouxi (which are closely related to C. segnis) was misidentified as C. segnis because they only differ in the distribution of the antennal sensilla and the shape of abdominal sclerites, characteristics that require dissection of the flies and preparation for microscopy (Delé colle 1985).

\section{On the specificity of haemosporidian-bird interactions}

Although Haemoproteus and Plasmodium parasites are capable of infecting birds from different families (Bensch et al. 2000; Beadell et al. 2004; Križanauskiené et al.
2006), Plasmodium is more likely to establish associations with novel hosts than Haemoproteus (Beadell et al. 2004). Supporting this possibility, Haemoproteus haplotypes recovered from birds belonging to the same families and superfamilies were generally grouped (see Fig. 2). For example, CulHae1 infected blue tits, great tits and eurasian nuthatches. Although both tits and nuthatches are included in different families, they are relatively close phylogenetically (Sibley \& Ahlquist 1990) and related species such as blue tits and great tits frequently share parasite haplotypes (Bensch et al. 2000). However, it may be the case that a similar ecological characteristic (hole nesting, in this case) could facilitate parasite hostswitching among tits and nuthatches. Furthermore, two Haemoproteus haplotypes FhHae1 (H. balmorali) and FhHae2 (H. pallidus) were isolated from pied flycatchers and clustered with other parasitic haplotypes isolated from birds belonging to the same family.

In support of the idea that Plasmodium has low specificity and a broad host range, the Plasmodium haplotype CulPlas1 infected birds belonging to different superfamilies (see Beadell et al. 2006 and Martinsen et al. 2006). Also, the Plasmodium haplotype SePlas1 isolated from eurasian nuthatches was grouped together with other haplotypes infecting species belonging to Passeroidea and Corvoidea superfamilies (Fig. 2).

\section{Presence of Plasmodium in biting midges}

Here, we isolated Plasmodium haplotypes from biting midges. However, isolation of parasite DNA from a biting midge does not imply transmission ability. In fact, with the exception of P. agamae (Petit et al. 1983), biting midges are not considered vectors of Plasmodium species. Previously, Haemoproteus haplotypes were isolated from mosquitoes (Ishtiaq et al. 2008; Njabo et al. 2011) although only biting midges and louse flies are known vectors of this parasite genus. The amplification of fragments of the parasite genome maintained in the insect after feeding and subsequent digestion of the bloodmeal could explain the molecular detection of Plasmodium DNA in parous biting midges. However, the digestive endonucleases of insects may fragment the DNA present in the bloodmeal, thus preventing the later amplification of large DNA fragments (Schall \& Smith 2006). Further experiments to establish patterns of transmission of malaria parasites are necessary to identify the potential role of biting midges as biological vectors.

Fig. 1 Bayesian inference of the biting midge morphospecies based on a cytochrome c oxidase subunit I gene fragment. The haplotypes isolated in the present study are marked in bold. Phylogenetic tree was obtained with the program MrBayes v3.2 using the substitution model HKY that was previously selected by means of jModeltest (see Materials and Methods). GenBank accession numbers are indicated between parentheses. Subgeneric position of the species is indicated after the accession numbers. 


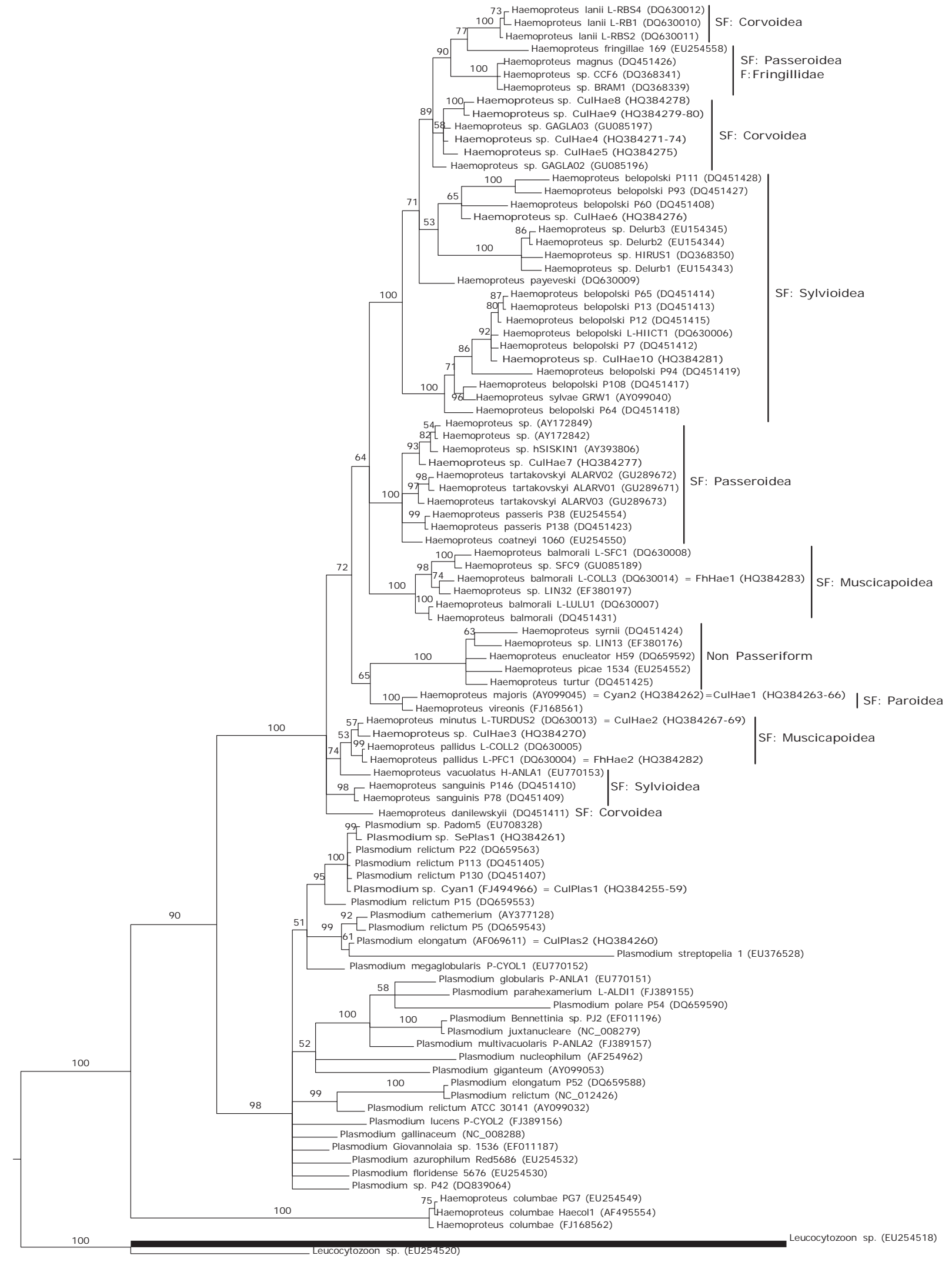

0.2 
Table 2 Results from ParaFit tests including Haemoproteus and Plasmodium haplotypes isolated from biting midges. Probabilities are computed after 999 random permutations. The null hypothesis of the global test is that parasites select hosts at random in the host phylogenetic tree. In the tests of individual hostparasite association links, the null hypothesis is that the link under test is random (indiscriminate). Only individual links with $\mathrm{P}<0.01$ were selected because the global test is not significant. Individual links with $\mathrm{P}<0.01$ are marked in bold

\begin{tabular}{|c|c|c|c|c|}
\hline Parasite & Host & P (BY) & P (ML) & $\mathrm{P}(\mathrm{NJ})$ \\
\hline GT & & 0.075 & 0.072 & 0.131 \\
\hline CulHae1 & $\mathrm{S} 1$ & 0.623 & 0.639 & 0.835 \\
\hline CulHae1 & S4 & 0.639 & 0.632 & 0.836 \\
\hline CulHae1 & C6 & 0.944 & 0.845 & 0.732 \\
\hline CulHae1 & K1 & 0.014 & 0.026 & 0.063 \\
\hline CulHae1 & K4 & 0.020 & 0.027 & 0.069 \\
\hline CulHae1 & $\mathrm{T} 1$ & 0.678 & 0.951 & 0.892 \\
\hline CulHae2 & S4 & 0.748 & 0.755 & 0.900 \\
\hline CulHae2 & K1 & 0.038 & 0.066 & 0.079 \\
\hline CulHae3 & K5 & 0.039 & 0.079 & 0.114 \\
\hline CulHae4 & S5 & 0.702 & 0.738 & 0.494 \\
\hline CulHae4 & S9 & 0.674 & 0.754 & 0.473 \\
\hline CulHae4 & C5 & 0.087 & 0.066 & 0.085 \\
\hline CulHae4 & FV10 & 0.008 & 0.002 & 0.009 \\
\hline CulHae5 & S4 & 0.728 & 0.765 & 0.617 \\
\hline CulHae7 & SG1 & 0.111 & 0.328 & 0.419 \\
\hline CulHae8 & S5 & 0.698 & 0.725 & 0.580 \\
\hline CulHae9 & S4 & 0.729 & 0.740 & 0.510 \\
\hline CulHae9 & S6 & 0.666 & 0.725 & 0.552 \\
\hline CulHae9 & $\mathrm{C} 2$ & 0.115 & 0.096 & 0.119 \\
\hline CulHae10 & FV11 & 0.007 & 0.003 & 0.001 \\
\hline CulHae10 & FV12 & 0.006 & 0.001 & 0.001 \\
\hline CulPlas1 & S5 & 0.511 & 0.465 & 0.475 \\
\hline CulPlas1 & S6 & 0.532 & 0.486 & 0.486 \\
\hline CulPlas1 & $\mathrm{K} 2$ & 0.594 & 0.707 & 0.762 \\
\hline CulPlas1 & $\mathrm{P} 1$ & 0.239 & 0.257 & 0.250 \\
\hline CulPlas1 & $\mathrm{T} 1$ & 0.043 & 0.051 & 0.137 \\
\hline
\end{tabular}

P, probability; BY, Bayesian inference; ML, maximum likelihood; NJ, neighbour joining; GT, global test.

On the specificity of haemosporidian-biting midge interactions

Using individual insects to identify specific associations between haplotypes of both insects and haemosporidians, we identified (i) the invertebrate hosts and parasites that have probably undergone cospeciation and (ii) the species most likely to have been subjected to hostswitching or sorting events (parasite extinction, or primary absence on daughter host lineage) (Legendre et al.
Table 3 Results from ParaFit tests including only Haemoproteus haplotypes isolated from biting midges. Probabilities are computed after 999 random permutations. The null hypothesis of the global test is that parasites select hosts at random in the host phylogenetic tree. In the tests of individual host-parasite association links, the null hypothesis is that the link under test is random (indiscriminate). Individual links with $\mathrm{P}<0.05$ are marked in bold

\begin{tabular}{|c|c|c|c|c|}
\hline Parasite & Host & P (BY) & P (ML) & $\mathrm{P}(\mathrm{NJ})$ \\
\hline GT & & 0.019 & 0.006 & 0.006 \\
\hline CulHae1 & S1 & 0.294 & 0.281 & 0.615 \\
\hline CulHae1 & $\mathrm{S} 4$ & 0.255 & 0.309 & 0.614 \\
\hline CulHae1 & C6 & 0.971 & 0.961 & 0.939 \\
\hline CulHae1 & K1 & 0.009 & 0.004 & 0.006 \\
\hline CulHae1 & K4 & 0.008 & 0.006 & 0.007 \\
\hline CulHae1 & $\mathrm{T} 1$ & 0.199 & 0.060 & 0.175 \\
\hline CulHae2 & $\mathrm{S} 4$ & 0.365 & 0.423 & 0.712 \\
\hline CulHae2 & K1 & 0.010 & 0.013 & 0.006 \\
\hline CulHae3 & K5 & 0.015 & 0.017 & 0.009 \\
\hline CulHae4 & S5 & 0.787 & 0.740 & 0.450 \\
\hline CulHae4 & S9 & 0.792 & 0.761 & 0.488 \\
\hline CulHae4 & C5 & 0.096 & 0.140 & 0.240 \\
\hline CulHae4 & FV10 & 0.014 & 0.007 & 0.022 \\
\hline CulHae5 & $\mathrm{S} 4$ & 0.829 & 0.779 & 0.539 \\
\hline CulHae7 & SG1 & 0.813 & 0.879 & 0.375 \\
\hline CulHae8 & S5 & 0.815 & 0.739 & 0.452 \\
\hline CulHae9 & $\mathrm{S} 4$ & 0.765 & 0.745 & 0.428 \\
\hline CulHae9 & S6 & 0.801 & 0.696 & 0.438 \\
\hline CulHae9 & $\mathrm{C} 2$ & 0.146 & 0.194 & 0.288 \\
\hline CulHae10 & FV11 & 0.001 & 0.005 & 0.001 \\
\hline CulHae10 & FV12 & 0.002 & 0.003 & 0.001 \\
\hline
\end{tabular}

P, probability; BY, Bayesian inference; ML, maximum likelihood; NJ, neighbour joining; GT, global test.

2002). Results including both Haemoproteus and Plasmodium haplotypes suggest that we are presumably dealing with a mixed coevolutionary structure with some random links added.

For Haemoproteus, biting midge haplotypes showed both specific and generalist relationships with parasite haplotypes. We found that some biting midges could act as generalist vectors with morphospecies such as C. simulator being involved in nine not statistically significant associations with Haemoproteus haplotypes. By contrast, some Haemoproteus haplotypes have probably undergone cospeciation with a single biting midge haplotype (K5-CulHae3, K1-CulHae2 and FV10-CulHae4), while others (CulHae1 and CulHae 10) undergone cospeciation with two different biting midge haplotypes.

Fig. 2 Bayesian inference of the haemosporidian haplotypes isolated from biting midges and birds using a partial sequence from the cytochrome B gene. The haplotypes isolated in this study are marked in bold. Phylogenetic tree was obtained with the program MrBayes v3.2 using the substitution model GTR that was previously selected by means of jModeltest (see Materials and Methods). GenBank accession numbers are indicated between parentheses. The superfamily (SF) of the bird species infected by the Haemoproteus haplotypes is indicated after the accession numbers. See Materials and Methods section for details of the analysis. 
(a)

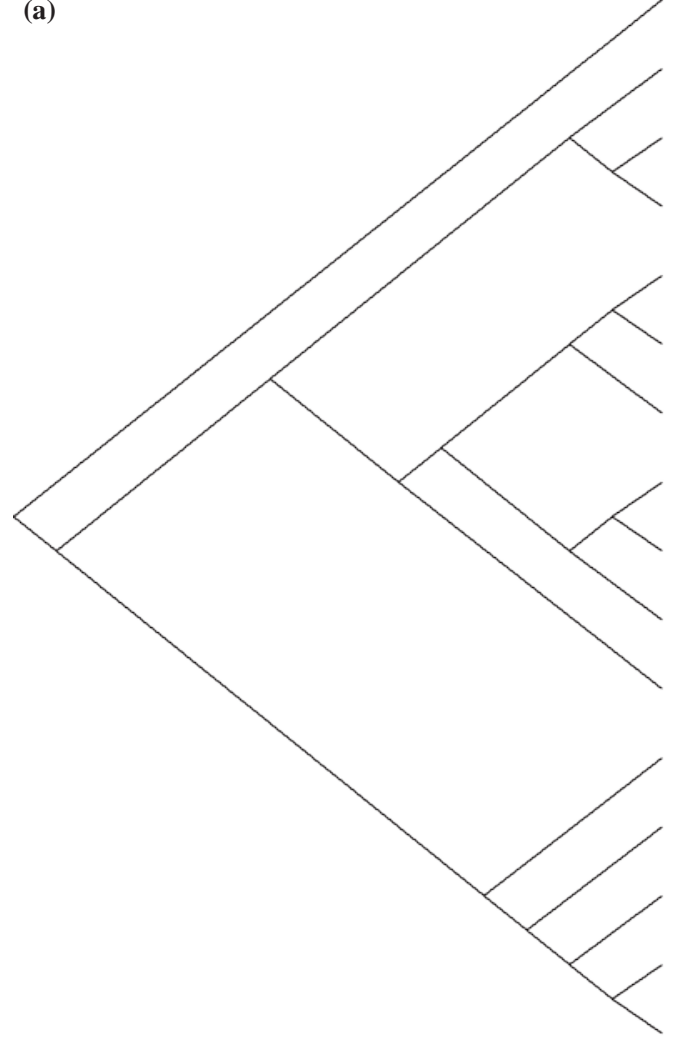

(b)
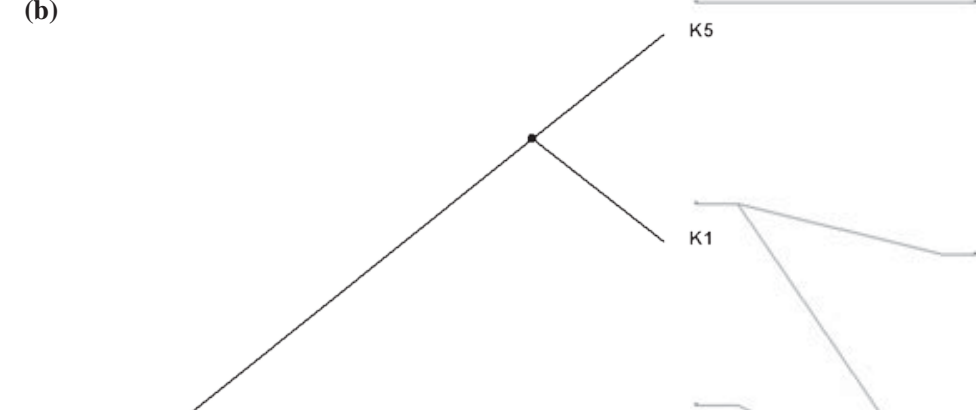

K5
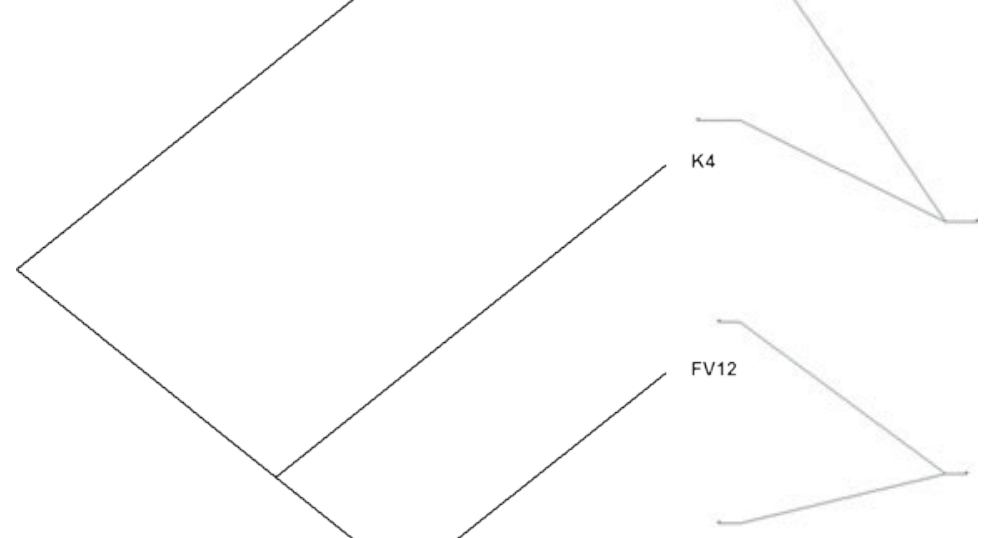

FV11

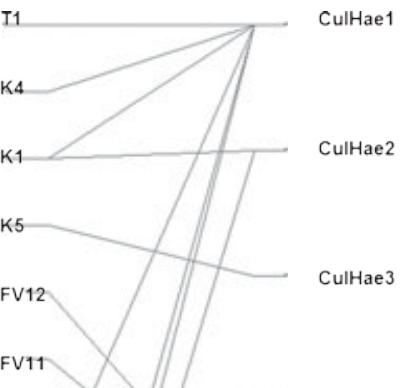

CulHae7
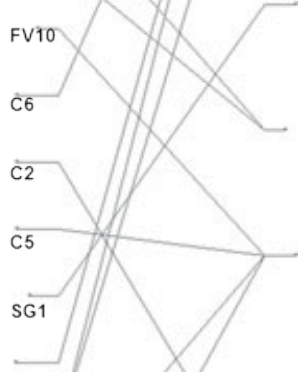

s1

$\mathrm{S4}$
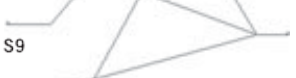

S6

s5

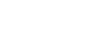

I

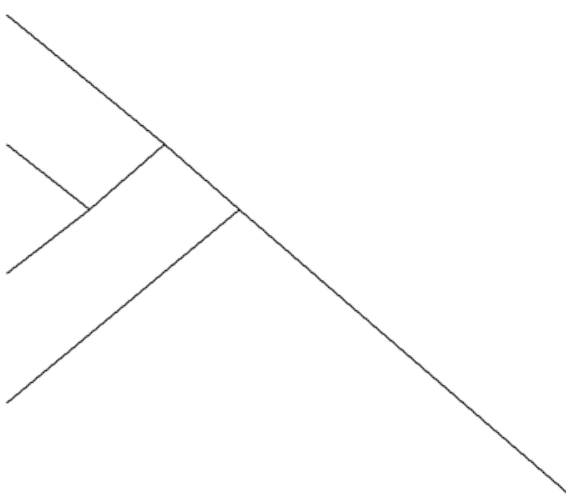

CulHae10

CulHae4

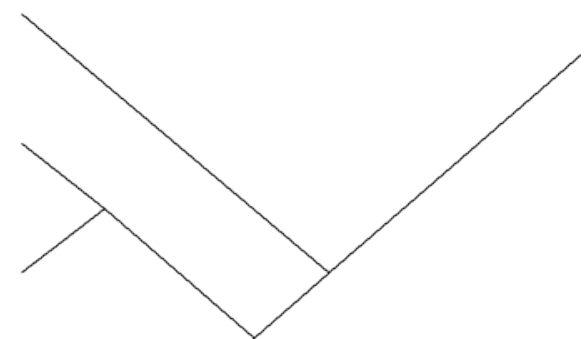

ulHae2

CulHae8

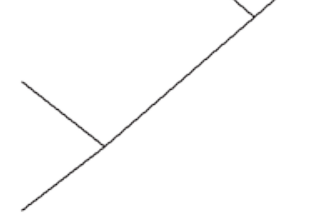

CulHae 3
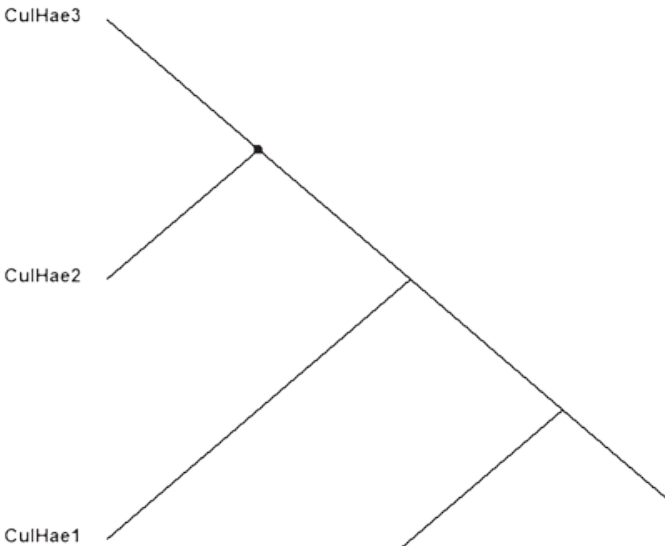

CulHae 10

CulHae1

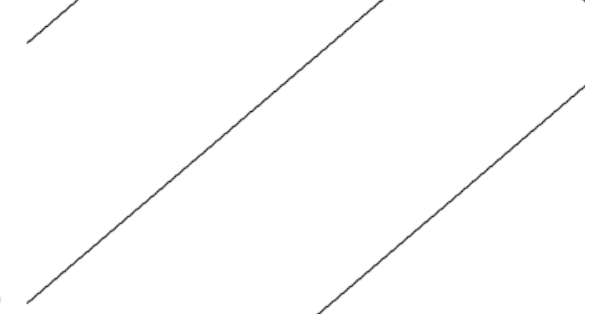

Fig. 3 Biting midges and Haemoproteus phylogenetic trees showing (a) all host-parasite links and (b) pruned trees showing the subset of haplotypes with significant associations using the ParaFit analysis. Maximum likelihood trees estimated for the hosts and parasites were used to perform the fit. Lines between trees depict the observed host-parasite associations. 
However, in the last cases, each blood parasite haplotype was found in biting midges haplotypes belonging to the same morphospecies and showing a low genetic distance between them $(<1 \%)$, supporting the difficulties of switching invertebrate hosts in these parasites (see Fig. 3b). It is well documented that successful development of blood parasites in vectors differs significantly between parasite-insect combinations (Ghosh et al. 2000; Habtewold et al. 2008). A successful infection of an invertebrate host probably implies the development of different mechanisms to avoid immune responses by insects. Although insect species in the same genus vary in their refractoriness to infection (Habtewold et al. 2008), responses against infections are probably more similar in closely related biting midge haplotypes (identified as the same morphospecies) and parasites could probably infect these haplotypes at a relative low adaptive cost (Combes 1997). Furthermore, the nonsignificant associations found between haplotypes CulHae 1, 2 and 4 with biting midge morphospecies other than C. kibunensis (haplotypes K1 and K4) and C. festivipennis (FV10; see Fig. 3a) may be a relatively recent attempt by the parasite to infect new invertebrate hosts which could finally render a cospeciation relationship.

Unlike the Haemoproteus parasites, we found only generalist relationships between biting midges and the three Plasmodium haplotypes detected. Thus, our results suggest that this parasite genus could be capable of exploiting a broad range of insects. Nevertheless, this study is about associations of parasites and insects, thus additional experiments to establish patterns of transmission are needed (see above). We agree with previous evidence supporting the fact that Plasmodium species were not tightly coevolved with vector species (Ishtiaq et al. 2008; Kimura et al. 2010; Njabo et al. 2011). As may be the case in the vertebrate host (Beadell et al. 2004), Plasmodium are probably more likely to establish associations with novel invertebrates than Haemoproteus. This may be specially the case of species such as P. relictum, a generalist parasite able to develop in more than 20 mosquito species belonging to different genera (Valkiūnas 2005), and was found in most of biting midge morphospecies included in this study (haplotype CulPlas1).

The test of a host-parasite link based on a small number of hosts, parasites and coevolutionary links may turn out not to be significant because of lack of power (Meier-Kolthoff et al. 2007). Sample sizes for some biting midge species in our study were low, and the hostparasite associations constituted by these species in our data set were not significant. On the contrary, significant coevolutionary links appear for species well represented in the data set, except for C. simulator whose haplotypes did not establish significant associations with any Haemoproteus haplotypes despite a similar sample size to that of C. festivipennis and C. kibunensis. Therefore, an increase in the sample size of some biting midge species could increase the number of coevolutionary host-parasite associations detected. However, it is difficult to obtain high sample sizes using natural populations to investigate parasite-vector-host interactions (see Hellgren et al. 2008).

\section{Acknowledgements}

This study was funded by projects CGL2006-14129-C02-01, CGL2007-61251 and CGL2009-09439 from MEC. The Junta de Castilla y León authorized the ringing and handling of birds. Javier Doné s (Director of 'Montes de Valsaín') gave permission to work in the study area. Currently, J.M.P. is supported by a JAE grant from C.S.I.C. We thank J. Moreno, G. Tomá s, E. Lobato, J. Morales, S. del Cerro, S. Ippi, Ó. Gordo and R. Ruiz-deCastañ eda for their help during fieldwork, four anonymous reviewers and Staffan Bensch for helpful comments and 'El Ventorrillo' field station for use of their facilities.

\section{References}

Beadell JS, Gering E, Austin J et al. (2004) Prevalence and differential host-specificity of two avian blood parasite genera in the Australo-Papuan region. Molecular Ecology, 13, 3829-3844.

Beadell JS, Ishtiaq F, Covas $\mathrm{R}$ et al. (2006) Global phylogeographic limits of Hawaii's avian malaria. Proceedings Biological Sciences, 273, 2935-2944.

Bensch S, Stjernman M, Hasselquist D et al. (2000) Host specificity in avian blood parasites: a study of Plasmodium and Haemoproteus mitochondrial DNA amplified from birds. Proceedings Biological Sciences, 267, 1583-1589.

Castresana J (2000) Selection of conserved blocks from multiple alignments for their use in phylogenetic analysis. Molecular Biology and Evolution, 17, 540-552.

Combes C (1997) Fitness of parasites: pathology and selection. International Journal for Parasitology, 21, 1-10.

Dallas JF, Cruickshank RH, Linton YM et al. (2003) Phylogenetic status and matrilineal structure of the biting midge, Culicoides imicola, in Portugal, Rhodes and Israel. Medical and Veterinary Entomology, 17, 379-387.

Delécolle JC (1985) Nouvelle contribution a l'étude systematique et iconographique des espèces du genre Culicoides (Diptera: Ceratopogonidae) du Nord-Est de la France. PhD Thesis, Universite Louis Pasteur de Strasbourg, 'Vie et Terre'.

Dyce AL (1969) The recognition of nulliparous and parous Culicoides (Diptera: Ceratopogonidae) without dissection. Journal of the Australian Entomological Society, 8, 11-15.

Ejiri H, Sato Y, Sasaki E et al. (2008) Detection of avian Plasmodium spp. DNA sequences from mosquitoes captured in Minami Daito Island of Japan. Journal of Veterinary Medical Science, 70, 1205-1210.

Ewald P (1994) The Evolution of Infectious Diseases. Oxford University Press, Oxford, UK, 320 p. 
Fallis AM, Wood DM (1957) Biting midges (Diptera: Ceratopogonidae) as intermediate hosts for Haemoproteus in ducks. Canadian Journal of Zoology, 35, 425-435.

Gager AB, del Rosario Loaiza J, Dearborn DC, Bermingham E (2008) Do mosquitoes filter the access of Plasmodium cytochrome b lineages to an avian host? Molecular Ecology, 17, 2552-2561.

Garnham PCC, Heisch RB, Minter DM, Phipps JD, Ikata M (1961) Culicoides adersi Ingram and Macfie, 1923, a presumed vector of Hepatocystis (= Plasmodium) kochi (Laveran, 1899). Nature, 190, 739-741.

Ghosh A, Edwards MJ, Jacobs-Lorena M (2000) The journey of the malaria parasite in the mosquito: hopes for the new century. Parasitology Today, 16, 196-201.

Habtewold T, Povelones M, Blagborough AM, Christophides GK (2008) Transmission blocking immunity in the malaria non-vector mosquito Anopheles quadriannulatus species A. PLoS Pathogens, 4, e1000070.

Hall TA (1999) BioEdit: a user-friendly biological sequence alignment editor and analysis program for Windows 95/98/NT. Nucleic Acids Symposium Series, 41, 95-98.

Hellgren O, Bensch S, Malmqvist B (2008) Bird hosts, blood parasites and their vectors-associations uncovered by molecular analyses of blackfly blood meals. Molecular Ecology, 17, 1605-1613.

Ishtiaq F, Guillaumot L, Clegg SM et al. (2008) Avian haematozoan parasites and their associations with mosquitoes across Southwest Pacific Islands. Molecular Ecology, 17, 4545-4555.

Kearn GC (1994) Evolutionary expansion of the Monogenea. International Journal of Parasitology, 24, 1227-1271.

Kim KS, Tsuda Y, Yamada A (2009) Bloodmeal identification and detection of avian malaria parasite from mosquitoes (Diptera: Culicidae) inhabiting coastal areas of Tokyo Bay, Japan. Journal of Medical Entomology, 46, 1230-1234.

Kimura M, Darbro JM, Harrington LC (2010) Avian malaria parasites share congeneric mosquito vectors. Journal of Parasitology, 96, 144-151.

Kremer M (1966) Contribution a l'etude du genre Culicoides Latreille particulierement en France. Encyclopedie d'Entomologie, Paris, serie A 39, 299 pp.

Križanauskiené A, Hellgren O, Kosarev V, Sokolov L, Bensch S, Valkiūnas G (2006) Variation in host specificity between species of avian hemosporidian parasites: evidence from parasite morphology and cytochrome $B$ gene sequences. The Journal of Parasitology, 92, 1319-1324.

Lambrechts L, Halbert J, Durand P, Gouagna LC, Koella JC (2005) Host genotype by parasite genotype interactions underlying the resistance of anopheline mosquitoes to Plasmodium falciparum. Malaria Journal, 4, 3.

Legendre P, Desdevises Y, Bazin E (2002) A statistical test for host-parasite coevolution. Systematic Biology, 51, 217-234.

Malmqvist B, Strasevicius D, Hellgren O, Adler PH, Bensch S (2004) Vertebrate host specificity of wild-caught blackflies revealed by mitochondrial DNA in blood. Biology Letters, 271, S152-S155.

Marquardt WC, Demaree RS, Grieve RB (2000) Parasitology and Vector Biology, 2nd edn. Academic Press, USA.

Martínez J, Martínez-de la Puente J, Herrero J et al. (2009) A restriction site to differentiate Plasmodium and Haemoproteus infections in birds: on the inefficiency of general primers for detection of mixed infections. Parasitology, 136, 713-722.

Martínez-de la Puente J, Merino S, Tomás G et al. (2010) The blood parasite Haemoproteus reduces survival in a wild bird: a medication experiment. Biology Letters, 6, 663-665.

Martinsen ES, Paperna I, Schall JJ (2006) Morphological versus molecular identification of avian Haemosporidia: an exploration of three species concepts. Parasitology, 133, 279-288.

Meier-Kolthoff JP, Auch AF, Huson DH, Göker M (2007) COPYCAT: co-phylogenetic analysis tool. Bioinformatics, 23, 898-900.

Merino S, Moreno J, Sanz JJ, Arriero E (2000) Are avian blood parasites pathogenic in the wild? A medication experiment in blue tits. Proceedings Biological Science, 267, 2507-2510.

Mullens BA, Cardona CJ, McClellan L, Szijj CE, Owen JP (2006) Culicoides bottimeri as a vector of Haemoproteus lophortyx to quail in California, USA. Veterinary Parasitology, 140, 35-43.

Nelson RL, Scrivani RP (1972) Isolations of arboviruses from parous midges of the Culicoides variipennis complex, and parous rates in biting populations. Journal of Medical Entomology, 9, 277-281.

Njabo KY, Cornel AJ, Bonneaud C et al. (2011) Nonspecific patterns of vector, host and avian malaria parasite associations in a central African rainforest. Molecular Ecology, 20, 1049-1061.

Nylander JA, Wilgenbusch JC, Warren DL, Swofford DL (2008) AWTY (Are We There Yet): a system for graphical exploration of MCMC convergence in Bayesian phylogenetics. Bioinformatics, 24, 581-583.

Petit G, Landau I, Boular Y, Gomes A, Touratier L (1983) Sporogonie de Plasmodium agamae chez Culicoides nubeculosus au laboratorie: I-Expérimentation et description du cycle. Protistologica, 19, 537-541.

Posada D (2008) ModelTest: phylogenetic model averaging. Molecular Biology and Evolution, 25, 1253-1256.

Poulin R (1992) Determinants of host specificity in parasites of freshwater fishes. International Journal of Parasitology, 22, 753758.

Rambaut A, Drummond AJ (2007) Tracer v1.4, Available from http://beast.bio.ed.ac.uk/Tracer.

Ronquist F, Huelsenbeck JP (2003) MrBayes 3: Bayesian phylogenetic inference under mixed models. Bioinformatics, 19, 1572-1574.

Schall JJ, Smith TC (2006) Detection of a malaria parasite (Plasmodium mexicanum) in ectoparasites (mites and ticks), and possible significance for transmission. Journal of Parasitology, 92, 413-415.

Sibley CG, Ahlquist JE (1990) Phylogeny and Classification of Birds: A Study in Molecular Evolution. Yale University Press, New Haven, CT.

Talavera G, Castresana J (2007) Improvement of phylogenies after removing divergent and ambiguously aligned blocks from protein sequence alignments. Systematic Biology, 56, 564-577.

Tamura K, Dudley J, Nei M, Kumar S (2007) MEGA4: molecular evolutionary genetics analysis (MEGA) software version 4.0. Molecular Biology and Evolution, 24, 1596-1599.

Tomás G, Merino S, Martínez-de la Puente J, Moreno J, Morales J, Lobato E (2008) A simple trapping method to 
estimate abundances of blood-sucking flying insects in avian nests. Animal Behaviour, 75, 723-729.

Valkiūnas G (2005) Avian Malaria Parasites and other Haemosporidia. CRC Press, Boca Raton, Florida, 932 p.

Valkiūnas G, Iezhova TA (2004) Detrimental effects of Haemoproteus infections on the biting midge Culicoides impunctatus (Diptera, Ceratopogonidae). Journal of Parasitology, 90, 194-196.

Valkiūnas G, Iezhova TA, Loiseau C, Sehgal RNM (2009) Nested cytochrome B polymerase chain reaction diagnostics detect sporozoites of hemosporidian parasites in peripheral blood of naturally infected birds. Journal of Parasitology, 95, 1512-1515.

Wilgenbusch JC, Warren DL, Swofford DL (2004) AWTY: a system for graphical exploration of MCMC convergence in Bayesian phylogenetic inference. Available from http:// ceb.csit.fsu.edu/awty.
J.M.-d.P. is interested in the study of interactions between insect vectors, wild birds and avian malaria parasites. J.M. is interested in the physiological effects of parasites on their vertebrate hosts and the evolution of host-parasite associations. J.R.-d.A. uses molecular tools to study avian immunological responses against parasite infections. J.H.'s research interests include parasite biology. S.M. is interested in understanding the ecology and evolution of host-parasite interactions.

\section{Data accessibility}

Sequences and TreeBase and GenBank nos. deposited at Dryad: doi:10.5061/dryad.9100. 